\title{
On the Role of the Environments and Star Formation for Quasar Activity
}

\author{
Daniela Bettoni ${ }^{1 *}$, Renato Falomo ${ }^{1}$, Jari K. Kotilainen ${ }^{2,3}$ and Kalle Karhunen ${ }^{3}$ \\ ${ }^{1}$ INAF - Osservatorio Astronomico di Padova, Padua, Italy, ${ }^{2}$ Finnish Centre for Astronomy with ESO (FINCA), University of \\ Turku, Turku, Finland, ${ }^{3}$ Tuorla Observatory, Department of Physics and Astronomy, University of Turku, Turku, Finland
}

\section{OPEN ACCESS}

Edited by: Deborah Dultzin

Universidad Nacional Autónoma de México, Mexico

Reviewed by:

Paolo Salucci,

Scuola Internazionale di Studi Superiori Avanzati (SISSA), Italy Zurab Silagadze, Budker Institute of Nuclear Physics (RAS), Russia

*Correspondence: Daniela Bettoni daniela.bettoni@oapd.inaf.it

Specialty section:

This article was submitted to

Cosmology,

a section of the journal

Frontiers in Astronomy and Space

Sciences

Received: 23 August 2017

Accepted: 31 October 2017

Published: 16 November 2017

Citation:

Bettoni D, Falomo R, Kotilainen JK and Karhunen K (2017) On the Role of the Environments and Star Formation for Quasar Activity.

Front. Astron. Space Sci. 4:44 doi: 10.3389/fspas.2017.00044
We investigate the host galaxy and environment properties of a sample of 400 low z $(<0.5)$ quasars that were imaged in the SDSS Stripe82. We can detect and study the properties of the host galaxy for more than $75 \%$ of the data sample. We discover that quasar are mainly hosted in luminous galaxies of absolute magnitude $M^{*}-3<M(R)<$ $M^{* 1}$ and that in the quasar environments the galaxy number density is comparable to that of inactive galaxies of similar luminosities. For these quasars we undertake also a study in $u, g, r, i$, and $z$ SDSS bands and again we discover that the mean colors of the quasar host galaxy it is not very different with respect to the values of the sample of inactive galaxies. For a subsample of low $z$ sources the imaging study is complemented by spectroscopy of quasar hosts and of close companion galaxies. This study suggests that the supply and cause of the nuclear activity depends only weakly on the local environment of quasars. Contrary to past suggestions, for low redshift quasar there is a very modest connection between recent star formation and the nuclear activity.

Keywords: galaxies active, galaxies evolution, galaxies nuclei, quasars general, quasars environment

\section{INTRODUCTION}

Quasar phenomenon assumes that the nuclear activity can be due to the major merger of two gasrich galaxies that feed the central engine and enable the growth of a spheroidal stellar component. However, important details on the mechanisms that triggers the gas fueling and how nuclear activity influence the successive evolution of the host galaxies remain poorly understood. The study of correlations among black hole masses, properties of the host galaxies and their environments may provide relevant clues to investigate the fundamental issues on quasar activity and its role on the evolution of galaxies. Simulations (e.g., Hopkins et al., 2013; Menci et al., 2014) suggest that minor and major merging events may have a key role for triggering and fueling the nuclear/quasar activity. These effects strictly depend on the global properties of the galaxy environment (Haehnelt and Kauffmann, 2000; Di Matteo et al., 2005).

The quasars, at low-redshift, follow the large-scale structure traced by galaxy clusters but they eschew the very centre of clusters (Söchting et al., 2002, 2004). On the other hand the quasar environment, on small scales (projected distance $<0.5 \mathrm{Mpc}$ ), appear overpopulated by blue disc galaxies having a significant star formation rate (Coldwell and Lambas, 2003, 2006). At higher redshifts, quasars are in some cases associated with richer environments (Hall and Green, 1998; Djorgovski, 1999) but also examples of modest galaxy environments are observed. The comparison of the environments of quasars, at $\mathrm{Mpc}$ scales, to those of galaxies has given contradictory results

${ }^{1} M^{*}$ and $L^{*}$ are the characteristic absolute magnitude and luminosity of the Schechter luminosity function of galaxies (Schechter, 1976). 
partially due to small samples and non homogeneous datasets. Early studies suggested that the galaxy environment of quasars is more strongly clustered than that of inactive galaxies (e.g., Shanks et al., 1988), while later studies based on surveys such as the Two Degree Field (2dF) and the Sloan Digital Sky Survey (SDSS) (Abazajian et al., 2009) found galaxy densities around quasars and inactive galaxies to be comparable to each other (e.g., Smith et al., 2000; Wake et al., 2004).

More recent studies using the SDSS archives such as Serber et al. (2006) and Strand et al. (2008) have taken advantage of the large datasets provided by the survey to study quasars at $z<0.4$. Both studies found that quasars are on average located in higher local over-density regions than typical $\mathrm{L}^{\star}$ galaxies, and that within $100 \mathrm{kpc}$ from the quasar the density enhancement is strongest. Serber et al. (2006) also claimed that: "high luminosity quasars have denser small-scale environments than those of lower luminosity." These studies are, however, limited by the deepness of the surveys that do not enable to study the galaxy population much fainter that $L^{\star}$. Meanwhile, a study by Bennert et al. (2008) found that many low-z quasars show signs of recent or on-going interactions with nearby galaxies, suggesting a connection between the environment and the quasar activation. In spite of these imaging studies our knowledge of the physical association of the galaxies around quasars and their dynamical properties remains uncertain.

To overcome these issues we recently carried out a systematic study of the the host galaxy and environment properties of a large $(\sim 400)$ sample of low redshift $(z<0.5)$ quasars based on the deep images available from the SDSS Stripe82 survey. These coadded images are $\sim 2$ magnitudes more profound with respect to the standard Sloan data and give the possibility to study both the quasar hosts and the immediate environments. We are able to resolve the host galaxy for $\sim 300$ targets (Falomo et al., 2014) and to investigate the the large scale galaxy environment of all quasars (Karhunen et al., 2014). For this work we adopt the concordance cosmology with $\mathrm{H}_{0}=70 \mathrm{~km} \mathrm{~s}^{-1} \mathrm{Mpc}^{-1}, \Omega_{m}=0.3$ and $\Omega_{\Lambda}=0.7$.

\section{THE SAMPLE AND ANALYSIS}

The sample of quasar we used, described in detail in Falomo et al. (2014), is derived from the fifth release of the SDSS Quasar Catalog (Schneider et al., 2010) that is based on the SDSS-DR7 data release (Abazajian et al., 2009). Our analysis is done only in the region of sky covered by the stripe 82 data, these images go deeper of about $\sim 2$ magnitudes with respect to the usual Sloan data and make possible the study of the quasar hosts, with these images we reach $m_{i \text { (limit) }}=24.1$ (Annis et al., 2014). The final sample is composed by 416 quasars in the range of redshift 0.1 $<z<0.5$. In this sample we are dominated by radio quiet quasars only 24 are radio loud (about $5 \%$ ). The mean redshift of the sample is $\langle z\rangle=0.39 \pm 0.08$ (median $0.41 \pm 0.06$ ) and the average absolute magnitude is $:\left\langle M_{i}\right\rangle=-22.68 \pm 0.61$ (median $-22.52 \pm 0.35$ ). To perform the analysis of the images we used the tool AIDA (Astronomical Image Decomposition and Analysis) (Uslenghi and Falomo, 2011). The tool is designed to perform 2D model fitting of quasar images including Adaptive Optics data and with detailed modeling of the PSF and its variations. In Figure 1 we show an example of our analysis and of the AIDA fit.

To perform the analysis of the large scale environment we used SExtractor (Bertin and Arnouts, 1996) to create our own galaxy catalogs for each image, measuring the magnitudes of the galaxies in each of the five filters separately. A comparison of the catalog generated with SExtractor to those of SDSS, and a visual inspection on a number of frames to further to study the validity of our classification is fully described in Karhunen et al. (2014). We found a good match between the SDSS and SExtractor catalogs at apparent magnitudes $m_{i}<23$, but at fainter magnitudes the number of objects detected by SExtractor dropped dramatically compared to those in the SDSS catalog. A visual inspection of these faint objects showed that they are mainly background noise which is either undetected by SExtractor or classified as an unknown object. We chose a conservative limit of classification value $\leq 0.20$ for our galaxies, to make sure we avoid all the stars and majority of the unknown objects.

\section{HOST GALAXIES}

For our sample we found that the morphology of the quasars host galaxy ranges from pure ellipticals to complex/composite morphologies that combine disks, spheroids, lens and halo and are dominated by luminous galaxies with absolute magnitude in the range $M^{*}-3<M(R)<M^{*}$ and the average absolute magnitude of this sample is $\left\langle M_{i}>=-22.68 \pm 0.61\right.$. The galaxy sizes (defined here as the half-light radius) range from very compact (few $\mathrm{kpc}$ ) up to more extended galaxies (10-15 $\mathrm{kpc}$ ) and, in our redshift range, not significant trend of change of the galaxy size with $\mathrm{z}$ is found. However, note that the sampled redshift range is small thus possibly hiding systematic changes of size with $\mathrm{z}$. It is interesting to note that the distribution of sizes of the quasars hosts is very similar to that of the sample of inactive galaxies of similar luminosity and redshift distribution as shown in Figure 2. The black hole mass of the quasar, estimated from the spectral properties of the nuclei, are poorly correlated with the total luminosity of the host galaxies but the relation between $\mathrm{M}_{B H}$ and the luminosity of the spheroidal component is consistent with that of local inactive galaxies (Falomo et al., 2014). We can resolve the host galaxy in $\sim 73 \%$ of the quasar sample but in u-band only for the $48 \%$.

Only the $(g-i)$ color is slighter bluer $(1.06 \pm 0.11)$ than that of inactive galaxies $(1.19 \pm 0.25)$ of similar luminosity and redshift (see Bettoni et al., 2015). The average absolute magnitude $\left(<M_{i}>_{\text {QSOHOST }}=-22.52<M_{i}>_{G A L}=-22.26\right)$ are very similar and also $(u-g)$ color of quasar hosts are similar $(1.40 \pm$ $0.30)$ to that of inactive galaxies $(1.54 \pm 0.62)$. Quasar and control sample have the same distribution of close ( $<50 \mathrm{kpc}$ projected) companion galaxies (Bettoni et al., 2015). This result is in contrast with that obtained by Matsuoka et al. (2014) who find similar (g-i) color for the quasar hosts but claim they are systematically bluer than the ensemble of normal galaxies in SDSS. Because of the different selection of active and non active galaxies of our and Matsuoka et al. (2014) samples it is not clear what is the cause of this discrepancy. 


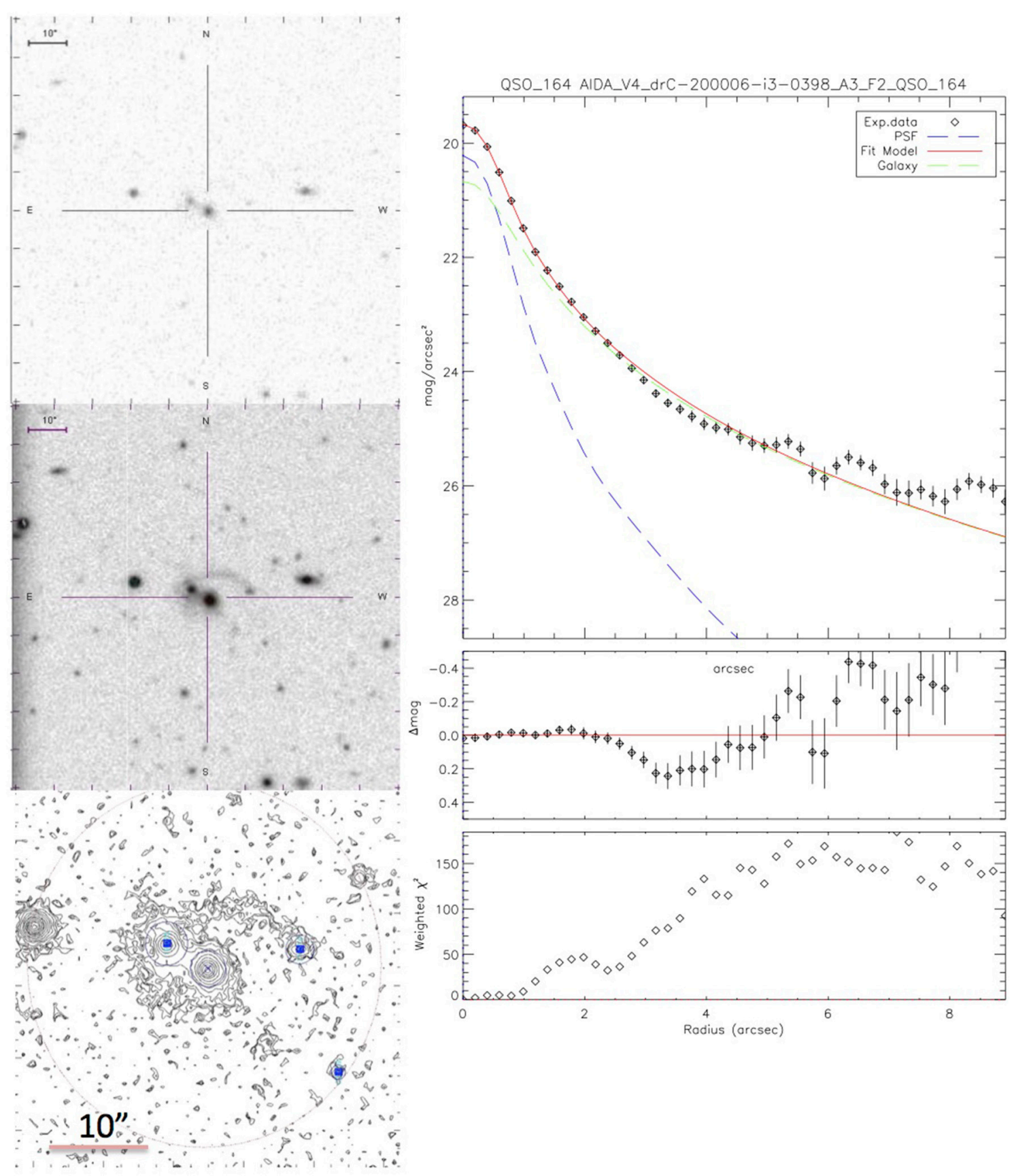

FIGURE 1 | Upper left: SDSS-DR7 i-band image, middle left: corresponding data from Stripe 82, bottom left: contour plot zoomed on the central object of the Stripe 82 i-band image (north at top, east left). Upper right: i-band radial profile together with the AIDA decomposition and fit. Middle right: distribution of residual for the best fit. Lower right: distribution of $\chi^{2}$.

\section{GALAXY ENVIRONMENT}

The large scale galaxy environment $(<1 \mathrm{Mpc})$ of the 302 resolved quasars compared with those of a sample of inactive galaxies at same redshift (Karhunen et al., 2014) shows that the galaxy number density is comparable to that of inactive galaxies with similar luminosities, as seen in Figure 3. For distances $<400$ $\mathrm{kpc}$ both quasars and inactive galaxies environments shows a significant excess compared to the background galaxy density. In particular the quasars, on average, have the tendency to be associated with small group of galaxies. No statistically significant difference is found between the over densities of low $\mathrm{z}$ quasar around the quasars and the inactive galaxies (Karhunen et al., 2014). No dependence of the over density on redshift, quasar 


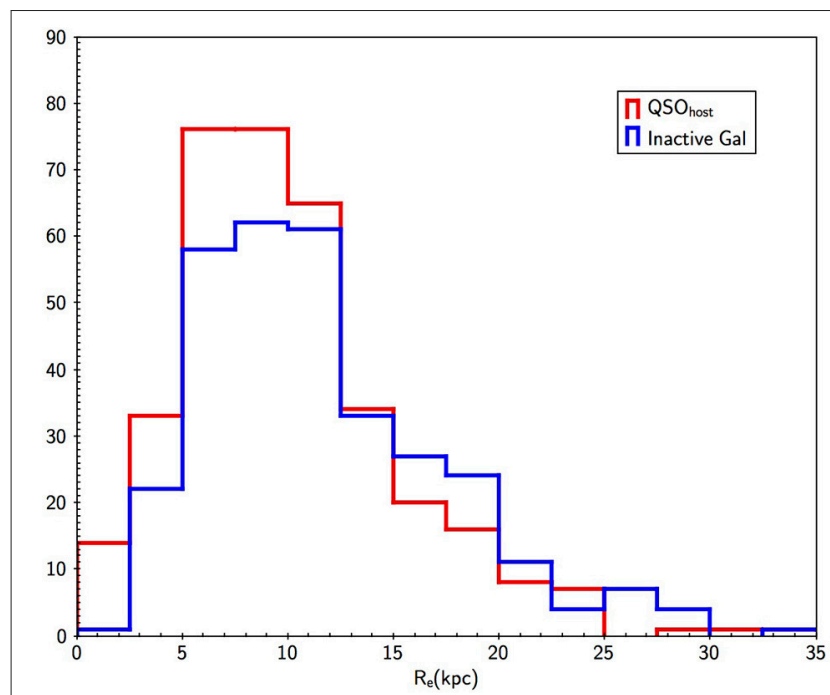

FIGURE 2 | Distribution of effective radius $R_{e}$ for quasar hosts (red line) and that of a sample of inactive galaxies (blue line) that have similar luminosity and redshift of quasar hosts.

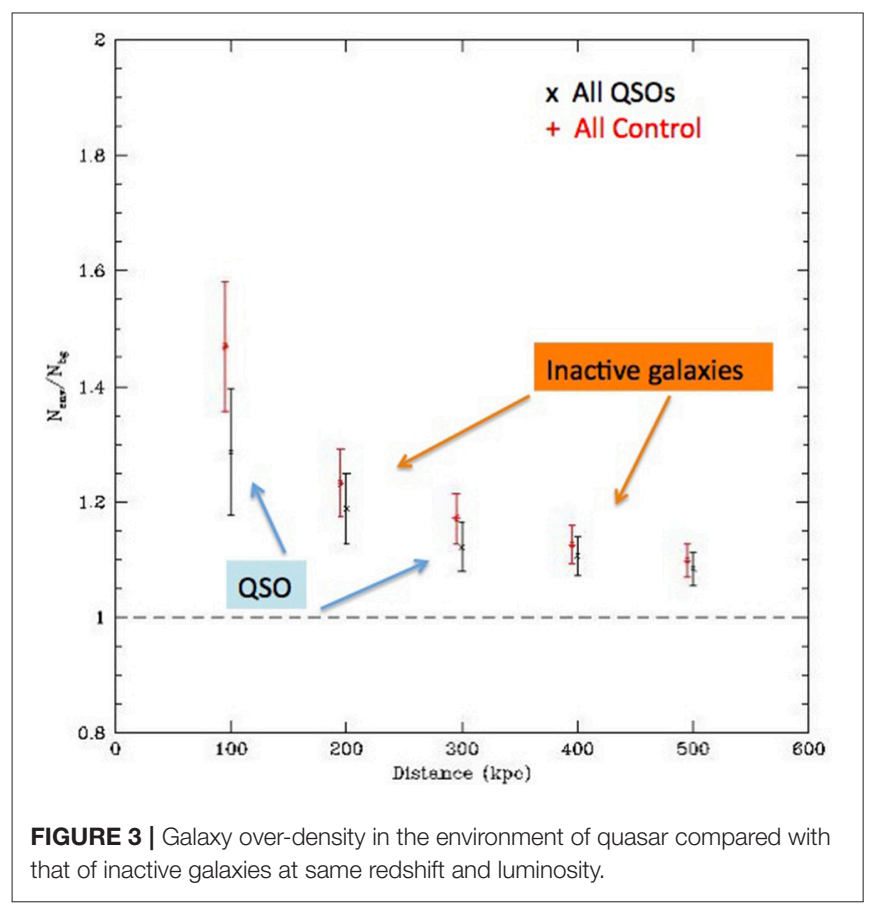

luminosity, the galaxies at same luminosity of the host galaxy, the radio luminosity or $\mathrm{BH}$ mass was found.

The lack of a notable difference between the quasars and nonactive galaxies (Karhunen et al., 2014) environments suggests that the connection between the quasar activity and its environment is less important than believed for fueling and activate the quasar. This may also indicate that secular evolution (e.g., disc instabilities, see; Ciotti et al., 2010) may play an important role in triggering the quasar activity, and that mergers are less important than expected.
As a second step in this environment study we used all five SDSS filters to analyze the color properties of the quasar environment (Karhunen et al. in preparation). This was done for a subsample of objects for which the SDSS-Stripe $82 \mathrm{u}$-band image was deep enough to allow the detection of nearby galaxies. This implies that we have to discard $\sim 20 \%$ of our original sample (typically u-band images with exposures $<40 \mathrm{~min}$ ). We find that the colors of the environments of the quasars are slightly bluer than those of inactive galaxies at all distances, with the effect being most noticeable in $\mathrm{g}-\mathrm{i}$. The color of the environment depends on the density of the environment, with denser areas being redder. We also find that quasars at higher redshifts have redder environments.

\subsection{Close Environments}

To better investigate, in all 5 Sloan bands, the very close environment of quasars in our sample, we selected both from the full sample of 416 quasar and from the comparison sample only objects at $z<0.3$ in fact, beyond this limit, the characterization of the quasar host galaxies becomes difficult at bluer filters due to the reduced contrast between the host galaxy and the nuclear emission. The detailed description of the final sample, composed by 52 quasars, is described in Bettoni et al. (2015). To compare the colors of the host galaxy we need to evaluate the K-correction for each filter, to this purpose we used the KCORRECT package (Blanton and Roweis, 2007). This allow us, using the luminosity+SED fitting, to derive also an estimate of the stellar mass for this subsample of quasar hosts and we used the stellar masses to derive the relation $\log M_{*}-\log M_{B H}$ shown in Figure 4. Our data are well fitted by the relation for nearby normal elliptical and S0 galaxies as derived by Reines and Volonteri (2015), confirming our findings that quasars are hosted in luminous early-type galaxies.

The two samples show similar properties in particular, for our sample of resolved objects, we find an average mass of the quasar host galaxy of $\left\langle M_{*}>=4.28 \pm 2.76 \times 10^{10} M_{\odot}\right.$ and $<M_{*}>=5.27 \pm 3.88 \times 10^{10} M_{\odot}$ for the comparison sample of normal galaxies. The overall mean colors of the quasar host galaxy are indistinguishable from those of inactive galaxies of similar luminosity and redshift. There is a suggestion that the most massive quasar hosts have bluer colors and show a higher star formation rate than those in the sample of inactive galaxies.

\subsection{Star Formation}

As a last step in our study we obtained with NOT+ALFOSC instrumentation long slit spectra of a subsample of the 52 quasar in Bettoni et al. (2015) and of the close companions (see details in Bettoni et al., 2017). We found that in 8 out of $12(\sim 67 \%)$ quasar the closest companion galaxy is associated to the quasar (same redshift). However the average level of star formation of companion galaxies that are associated with the quasar appears similar to that of the companion galaxies that are not associated with the quasar. These results suggest a modest role of the quasar emission for the SF in nearby companion galaxies. Finally for 3 targets we observed also the spectrum of the host galaxy which turned out to be typical of an old stellar population. 


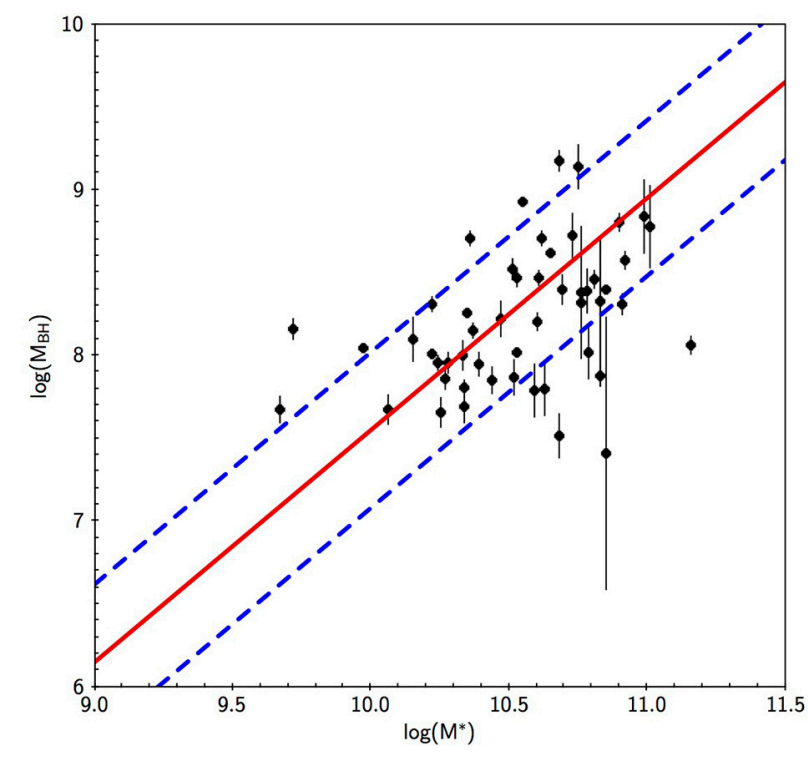

FIGURE 4 | Correlation between quasar virial black hole mass and the total stellar mass of their host galaxies as derived from SED fitting. Despite the large scatter the relation is similar to that obtained by Reines and Volonteri (2015) for inactive galaxies and bulges (red solid line). The $1 \sigma$ uncertainties are indicated by blue dashed lines.

\section{CONCLUSIONS}

From our multi-band study of a large sample of nearby quasar we found that:

Quasar host galaxy luminosity is mainly in the range $M^{*}-3<$ $M_{R}<M^{*}$.

Galaxy environments of quasar are similar to those of a sample of inactive galaxies at similar redshift and luminosity. The colors of the galaxies in the immediate environments of the quasars are slightly bluer than those of inactive galaxies at all distances; the effect being most noticeable in $(g-i)$ color.

Overall the mean colors $(g-i=0.82 \pm 0.26$; $r-i=0.26$ \pm 0.16 and $u-g=1.32 \pm 0.25$ ) of the quasar host galaxy are indistinguishable from those of inactive galaxies of similar luminosity and redshift.

In $\sim 50 \%$ of the quasar in the $\mathrm{z}<0.3$ sample, we found companion galaxies at projected distance $<50 \mathrm{kpc}$ that could be associated to the quasar.

Optical spectroscopy of a subsample of 12 quasar shows that these associated companions exhibit [OII] $\lambda$ 3,727 $\AA$ emission

\section{REFERENCES}

Abazajian, K. N., Adelman-McCarthy, J. K., Agüeros, M. A., Allam, S. S., Allende Prieto, C., An, D., et al. (2009). The seventh data release of the sloan digital sky survey. Astrophys. J. Suppl. 182, 543-558. doi: 10.1088/0067-0049/182/2/543

Annis, J., Soares-Santos, M., Strauss, M. A., Becker, A. C., Dodelson, S., Fan, X., et al. (2014). The sloan digital sky survey coadd: $275 \mathrm{deg}^{2}$ of lines suggestive of episodes of (recent) star formation possibly induced by past interactions. The rate of star formation is, however, similar to that of companions of inactive galaxies. This may indicate that the presence of the quasar do not change the star formation in the associated close companions. Contrary to past suggestions (e.g., Matsuoka et al., 2014) the role of these associated companion galaxies for triggering and fueling the nuclear activity appears thus marginal as similar conditions are observed in inactive galaxies of mass similar to that of quasar hosts. Contrary to past suggestions. A significant time delay between the phase of nuclear activity and the star formation in the immediate environments could smear the link between them.

\section{AUTHOR CONTRIBUTIONS}

DB and RF wrote the paper and analyzed the data JK helped in the selection of the sample and the discussion of the results and KK made the NOT observations. All authors participated in the discussion of the final results.

\section{ACKNOWLEDGMENTS}

Funding for the SDSS and SDSS-II has been provided by the Alfred P. Sloan Foundation, the Participating Institutions, the National Science Foundation, the U.S. Department of Energy, the National Aeronautics and Space Administration, the Japanese Monbukagakusho, the Max Planck Society, and the Higher Education Funding Council for England. The SDSS Web Site is http://www.sdss.org/.

The SDSS is managed by the Astrophysical Research Consortium for the Participating Institutions. The Participating Institutions are the American Museum of Natural History, Astrophysical Institute Potsdam, University of Basel, University of Cambridge, Case Western Reserve University, University of Chicago, Drexel University, Fermilab, the Institute for Advanced Study, the Japan Participation Group, Johns Hopkins University, the Joint Institute for Nuclear Astrophysics, the Kavli Institute for Particle Astrophysics and Cosmology, the Korean Scientist Group, the Chinese Academy of Sciences (LAMOST), Los Alamos National Laboratory, the Max-PlanckInstitute for Astronomy (MPIA), the Max-Planck-Institute for Astrophysics (MPA), New Mexico State University, Ohio State University, University of Pittsburgh, University of Portsmouth, Princeton University, the United States Naval Observatory, and the University of Washington. doi: 10.1088/0004-637X/794/2/120

Bennert, N., Canalizo, G., Jungwiert, B., Stockton, A., Schweizer, F., Peng, C. Y., et al. (2008). Evidence for merger remnants in early-type host galaxies of low-redshift QSOs. Astrophys. J. 677, 846-857. doi: 10.1086/529068

Bertin, E., and Arnouts, S. (1996). SExtractor: Software for source extraction. Astron. Astrophys. Suppl. 117, 393-404. doi: 10.1051/aas:1996164 
Bettoni, D., Falomo, R., Kotilainen, J. K., and Karhunen, K. (2017). Lowredshift quasars in the SDSS Stripe 82: associated companion galaxies and signature of star formation. Month. Notices R. Astron. Soc. 466, 3600-3611. doi: $10.1093 / \mathrm{mnras} / \mathrm{stw} 3284$

Bettoni, D., Falomo, R., Kotilainen, J. K., Karhunen, K., and Uslenghi, M. (2015). Low-redshift quasars in the SDSS Stripe 82. Host galaxy colours and close environment. Month. Notices R. Astron. Soc. 454, 4103-4113. doi: $10.1093 / \mathrm{mnras} / \mathrm{stv} 2233$

Blanton, M. R., and Roweis, S. (2007). K-corrections and filter transformations in the ultraviolet, optical, and near-infrared. Astron. J. 133, 734-754. doi: 10.1086/510127

Ciotti, L., Ostriker, J. P., and Proga, D. (2010). Feedback from central black holes in elliptical galaxies. III. models with both radiative and mechanical feedback. Astrophys. J. 717, 708-723. doi: 10.1088/0004-637X/717/2/708

Coldwell, G. V., and Lambas, D. G. (2003). Star formation in quasar and active galaxy environments. Month. Notices R. Astron. Soc. 344, 156-160. doi: 10.1046/j.1365-8711.2003.06804.x

Coldwell, G. V., and Lambas, D. G. (2006). Properties of galaxies in Sloan Digital Sky Survey quasar environments at $\mathrm{z}<0.2$. Month. Notices R. Astron. Soc. 371, 786-792. doi: 10.1111/j.1365-2966.2006.10712.x

Di Matteo, T., Springel, V., and Hernquist, L. (2005). Energy input from quasars regulates the growth and activity of black holes and their host galaxies. Nature 433, 604-607. doi: 10.1038/nature03335

Djorgovski, G. (1999). “GRB host galaxies,” KITP Conference: Gamma Ray Bursts and their Afterglows (Santa Barbara, CA).

Falomo, R., Bettoni, D., Karhunen, K., Kotilainen, J. K., and Uslenghi, M. (2014). Low-redshift quasars in the Sloan Digital Sky Survey Stripe 82. The host galaxies. Month. Notices R. Astron. Soc. 440, 476-493. doi: $10.1093 / \mathrm{mnras} / \mathrm{stu} 283$

Haehnelt, M. G., and Kauffmann, G. (2000). The correlation between black hole mass and bulge velocity dispersion in hierarchical galaxy formation models. Month. Notices R. Astron. Soc. 318, L35-L38. doi: 10.1046/j.1365-8711.2000.03989.x

Hall, P. B., and Green, R. F. (1998). An optical/near-infrared study of radioloud quasar environments. II. Imaging results. Astrophys. J. 507, 558-584. doi: $10.1086 / 306349$

Hopkins, P. F., Cox, T. J., Hernquist, L., Narayanan, D., Hayward, C. C., and Murray, N. (2013). Star formation in galaxy mergers with realistic models of stellar feedback and the interstellar medium. Month. Notices R. Astron. Soc. 430, 1901-1927. doi: 10.1093/mnras/stt017

Karhunen, K., Kotilainen, J. K., Falomo, R., and Bettoni, D. (2014). Low-redshift quasars in the SDSS Stripe 82. The local environments. Month. Notices $R$. Astron. Soc. 441, 1802-1816. doi: 10.1093/mnras/stu688

Matsuoka, Y., Strauss, M. A., Price, III, T. N., and DiDonato, M. S. (2014). Massive star-forming host galaxies of quasars on sloan digital sky survey stripe 82 . Astrophys. J. 780:162. doi: 10.1088/0004-637X/780/2/162
Menci, N., Gatti, M., Fiore, F., and Lamastra, A. (2014). Triggering active galactic nuclei in hierarchical galaxy formation: disk instability vs. interactions. Astron. Astrophys. 569:A37. doi: 10.1051/0004-6361/201424217

Reines, A. E., and Volonteri, M. (2015). Relations between central black hole mass and total galaxy stellar mass in the local universe. Astrophys. J. 813:82. doi: 10.1088/0004-637X/813/2/82

Schechter, P. (1976). An analytic expression for the luminosity function for galaxies. Astrophys. J. 203, 297-306. doi: 10.1086/154079

Schneider, D. P., Richards, G. T., Hall, P. B., Strauss, M. A., Anderson, S. F., Boroson, T. A., et al. (2010). The sloan digital sky survey quasar catalog. V. seventh data release. Astron. J. 139:2360. doi: 10.1088/0004-6256/139/6/2360

Serber, W., Bahcall, N., Ménard, B., and Richards, G. (2006). The smallscale environment of quasars. Astrophys. J. 643, 68-74. doi: 10.1086/ 501443

Shanks, T., Boyle, B. J., and Peterson, B. A. (1988). “The spatial clustering of QSO's," in Optical Surveys for Quasars, Vol. 2 of Astronomical Society of the Pacific Conference Series (San Francisco, CA), 244.

Smith, R. J., Boyle, B. J., and Maddox, S. J. (2000). The environments of intermediate-redshift QSOs: $0.3<\mathrm{z}<0.7$. Month. Notices R. Astron. Soc. 313, 252-262. doi: 10.1046/j.1365-8711.2000.03225.x

Söchting, I. K., Clowes, R. G., and Campusano, L. E. (2002). Quasar environment in the context of large-scale structure at $\mathrm{z} \sim 0.3$. Month. Notices R. Astron. Soc. 331, 569-577. doi: 10.1046/j.1365-8711.2002.05123.x

Söchting, I. K., Clowes, R. G., and Campusano, L. E. (2004). Relation of radioquiet quasars to galaxy clusters at $\mathrm{z}<0.3$. Month. Notices R. Astron. Soc. 347 , 1241-1254. doi: 10.1111/j.1365-2966.2004.07306.x

Strand, N. E., Brunner, R. J., and Myers, A. D. (2008). AGN environments in the sloan digital sky survey. I. dependence on type, redshift, and luminosity. Astrophys. J. 688, 180-189. doi: 10.1086/592099

Uslenghi, M., and Falomo, R. (2011). AIDA: a software package for 2D model fitting analysis of astronomical images. Appl. Digital Image Process. XXXIV, 8135:813524. doi: 10.1117/12.913305

Wake, D. A., Miller, C. J., Di Matteo, T., Nichol, R. C., Pope, A., Szalay, A. S., et al. (2004). The clustering of active galactic nuclei in the sloan digital sky survey. Astrophys. J. Lett. 610, L85-L88. doi: 10.1086/423317

Conflict of Interest Statement: The authors declare that the research was conducted in the absence of any commercial or financial relationships that could be construed as a potential conflict of interest.

Copyright (c) 2017 Bettoni, Falomo, Kotilainen and Karhunen. This is an open-access article distributed under the terms of the Creative Commons Attribution License (CC $B Y)$. The use, distribution or reproduction in other forums is permitted, provided the original author(s) or licensor are credited and that the original publication in this journal is cited, in accordance with accepted academic practice. No use, distribution or reproduction is permitted which does not comply with these terms. 